This item was submitted to Loughborough's Research Repository by the author.

Items in Figshare are protected by copyright, with all rights reserved, unless otherwise indicated.

\title{
Contrasting effects of magnetic field on thermodynamic and resistive transitions in high- Tc cuprates
}

PLEASE CITE THE PUBLISHED VERSION

PUBLISHER

(C) American Physical Society

LICENCE

CC BY-NC-ND 4.0

REPOSITORY RECORD

Alexandrov, A.S., W.H. Beere, V.V. Kabanov, and W.Y. Liang. 2019. "Contrasting Effects of Magnetic Field on Thermodynamic and Resistive Transitions in High- Tc Cuprates”. figshare. https://hdl.handle.net/2134/1317. 


\title{
Contrasting Effects of Magnetic Field on Thermodynamic and Resistive Transitions in High- $T_{c}$ Cuprates
}

\author{
A. S. Alexandrov, ${ }^{1}$ W. H. Beere, ${ }^{2, *}$ V. V. Kabanov, ${ }^{3}$ and W. Y. Liang ${ }^{2}$ \\ ${ }^{1}$ Department of Physics, Loughborough University, Loughborough LE11 3TU, United Kingdom \\ ${ }^{2}$ Interdisciplinary Research Centre in Superconductivity, University of Cambridge, Cambridge CB3 OHE, United Kingdom \\ ${ }^{3}$ Frank Laboratory of Neutron Physics, Joint Institute for Nuclear Research, Dubna, Russia
}

(Received 24 February 1997)

\begin{abstract}
For a number of high- $T_{c}$ cuprates it has been observed that the resistive transition reveals an upper critical field with increasingly negative gradient on cooling. However, the $\lambda$ point of the specific heat scarcely shifts with applied magnetic field. Either phenomenon is highly unusual in itself, but also appears to be irreconcilable with one another under the BCS framework. This Letter offers an explanation of the observed phenomena on the basis of the bipolaron theory. [S0031-9007(97)03885-4]
\end{abstract}

PACS numbers: 74.20. $-\mathrm{z}, 67.20 .+\mathrm{k}$

There are several strikingly universal properties of high- $T_{c}$ cuprates, in particular, their critical phenomena. It has been established beyond doubt [1] that in high- $T_{c}$ superconductors the anomaly in the specific heat spreads to about $\left|T-T_{c}\right| / T_{c} \sim 0.1$ or larger. The estimations with the canonical Gaussian fluctuations yield an unusually small coherence volume, comparable or even less than the unit cell volume [2]. That means that the overlap of pairs is small. Another striking critical phenomenon of cuprates is their unusually high values of the upper critical field, and its temperature dependence. In many superconducting oxides, including the cubic ones, an upward curvature of $H_{c 2}(T)$ has been observed ([3-8] and references therein). There have been several attempts to explain the divergent resistive $H_{c 2}(T)$ on cooling (see, for example, [7] and [9]). However, only the small bipolaron theory [10] with pairs preformed above $T_{c}$ has successfully predicted this phenomenon [11]. Further evidence for the charged $2 e$ Bose liquid was provided by the quantitative mapping of the specific heat of several high- $T_{c}$ cuprates in zero magnetic field to the $\lambda$ curve of $\mathrm{He}^{4}$ [12]. Also the in-plane and $c$ axes transport as well as the normal state gap observed in the NMR [13], the infrared reflectivity [14], and the angle-resolved photoemission [15], all find a satisfactory explanation in the preformed boson model [16-19].

In this Letter, by applying the Alexandrov-Mott theory [10], we explain a startling behavior of the thermodynamic phase transition in a magnetic field [1,20-22] contrasting with the resistive transition [3-8]. Our approach to the critical phenomena of high- $T_{c}$ superconductors emphasizes their striking universality being independent of the coupling mechanism and the symmetry of the order parameter.

In a BCS superconductor there is a well defined step in the specific heat at the transition temperature; this transition temperature tracks the sharp resistive transition in all magnetic fields. For most high- $T_{c}$ cuprates in zero magnetic field, there is also a well defined $\lambda$ type peak in the specific heat at the resistive transition temperature.
However, as shown in the comprehensive experimental study by Junod et al. [20] and by several other groups $[1,21,22]$ the effect of magnetic field on the specific heat anomaly is to reduce the peak height but leave its position largely unchanged. The peak in specific heat is usually associated with critical fluctuations. This view point is reinforced when the behavior is compared with that of liquid helium-4 [12]. On the other hand, the resistive transition shows practically parallel shift towards lower temperatures in the Lorentz-force free geometry [7]. Consequently in high- $T_{c}$ cuprates, the resistive behavior does not correspond to that of the specific heat, except for zero magnetic field. Moreover, applying the canonical fluctuation theory based on the Ginsburg-Landau (GL) free energy one arrives at a quite meaningless value of the zero temperature coherence length, which appears to be of the same order as the wavelength of holes or even less, hence invalidating the main assumption of the GL and BCS theory [23].

We argue that the superfluid transition temperature in cuprates is given by the resistive measurements and that the specific heat anomaly owes its origin to some other phenomenon. In the theory of critical phenomena the peak in the specific heat is associated with the diverging correlation length as the transition is approached. With the introduction of a magnetic field some of the long range order is suppressed; this reduces the superfluid transition temperature as measured by resistivity. The specific heat anomaly, however, remains largely unaffected by the magnetic field as it is governed by short range effects (see also discussion in Ref. [22]). Only the peak height is reduced because of the suppression of the long range order by the magnetic field. We therefore regard the specific heat anomaly as mainly due to short range fluctuations, and the temperature at which these occur practically unaffected by the application of a magnetic field.

In the following we show that a weakly interacting charged Bose gas (CBG) in a magnetic field has precisely the property which reproduces both the specific heat and the resistive transition of cuprates. In a Bose system the 
value of the chemical potential is governed by the total density sum rule,

$$
n_{B}=\int d \epsilon \rho(\epsilon) f(\epsilon),
$$

where $\rho(\epsilon)$ is the density of states and $f(\epsilon)$ is the BoseEinstein distribution function,

$$
f(\epsilon)=\frac{1}{\exp [(\epsilon-\mu) / T]-1} .
$$

The specific heat can be calculated from the derivative of the energy,

$$
C(T, H)=\frac{d}{d T} \int d \epsilon \frac{\rho(\epsilon) \epsilon}{\exp [(\epsilon-\mu) / T]-1} .
$$

The result is

$$
C(T, H)=\frac{\left\langle\epsilon^{2}\right\rangle}{T^{2}}-\frac{\langle\epsilon\rangle^{2}}{T^{2}\langle 1\rangle},
$$

where

$$
\left\langle\epsilon^{x}\right\rangle=-T \int d \epsilon \rho(\epsilon) \frac{\partial f(\epsilon)}{\partial \epsilon} \epsilon^{x} .
$$

We note that the specific heat calculated from Eq. (4) is invariant to a shift in the energy, $\epsilon \rightarrow \epsilon+\epsilon_{0}$, as it should be $\left(\hbar=c=k_{B}=1\right)$.

For a system of ideal bosons in 3 dimensions the density of states (DOS) is given by

$$
\rho(\epsilon)=\frac{m^{3 / 2} \sqrt{\epsilon}}{\sqrt{2} \pi^{2}},
$$

where $m=\left(m_{a b}^{2} m_{c}\right)^{1 / 3}$ with $m_{a b}$ and $m_{c}$ being the inplane and $c$-axis effective masses, respectively. With magnetic field perpendicular to the planes the bosons are quantized into Landau levels with the associated DOS,

$$
\rho(\epsilon, H)=\frac{m^{3 / 2} \omega_{H}}{2 \sqrt{2} \pi^{2}} \sum_{n=0}^{\infty}\left[\epsilon-\omega_{H}\left(n+\frac{1}{2}\right)\right]^{-1 / 2},
$$

where $\omega_{H}=2 \mathrm{eH} / \mathrm{m}_{a b}$ is the cyclotron frequency.

The difference between the effect of the magnetic field on resistivity and on the specific heat becomes transparent if we calculate the specific heat in the presence of the magnetic field. For an ideal CBG the Landau energy levels are essentially one dimensional. Thus there is no Bose-Einstein condensation in a magnetic field [24], and the resistive transition is immediately suppressed such that $T_{c}(H)=0$ for all $H$. We deliberately retain the features of the noninteracting charged Bose gas which prevents Bose-Einstein condensation to investigate the behavior of the specific heat in the absence of a condensate. This is to test our premise that the specific heat in a finite magnetic field is unrelated to the condensate formation.

As can be seen from Eq. (4) the form of the specific heat depends on various integrals over the density of states. The main weight of these integrals sits on either the upper or lower limit, depending on the exponent in $\epsilon^{x}$. We are considering the limit of high temperatures, so $T \gg \omega_{H}$. Only the lowest Landau level can produce a divergent integral (for $\mu \rightarrow \omega_{H} / 2$ ), the effect of all the other Landau levels is much less sensitive to the DOS shape. Nevertheless, to be precise we perform the numerical calculations of the chemical potential $\mu$, Eq. (1), and the specific heat, Eq. (5), with the exact DOS, Eq. (7). The results for several relative values of the magnetic field are presented in Figs. 1 and 2. The characteristic temperature at which the chemical potential changes its slope remains about $T_{c 0}=3.31 n_{B}^{2 / 3} / m$ as long as $\omega_{H} \ll T_{c 0}$. Only in a very strong field, $\omega_{H} \simeq T_{c 0}$, Fig. 1 , this characteristic temperature shifts appreciably towards zero. Therefore, the position of the specific heat anomaly remains largely unaffected by the moderate magnetic field, Fig. 2. The suppression of the maximum value of $C(T, H)$ goes as the square root of the magnetic field, thus even a moderate magnetic field can produce a sizable change in the value of the specific heat at $T=T_{c 0}$ without any appreciable change in the peak position. Both results reproduce fairly well the experimental data by Junod et al. [20]. On a quantitative level one can compare the theoretical change in the specific heat $C(T, H)-C\left(T, H_{1}\right)$ with the experimental one thus eliminating all field-independent contributions, e.g., the lattice specific heat. A parameterfree fit to the experimental data for $\mathrm{Hg}-1223$ [21] is shown in Fig. 3 with $H_{1}=7 T$ and the in-plane bipolaron mass $m_{a b}=17.1 m_{e}$. The effective mass and the number of bosons $n_{B}$ affect only the absolute values of $C(T, H)-C\left(T, H_{1}\right)$ and $C(T, H)$ with a little effect on the anomaly shape [25]. One could hardly expect that our simple model would be able to describe quantitatively the critical fluctuation effects which are responsible for the shape of the specific heat near the $\lambda$ point. Nevertheless, the result is that the theoretical shape agrees well with the experimental evidence, Fig. 3. Hence the weakly interacting CBG appears to be a fair approximation for the ground state of high- $T_{c}$ cuprates. We believe that a large

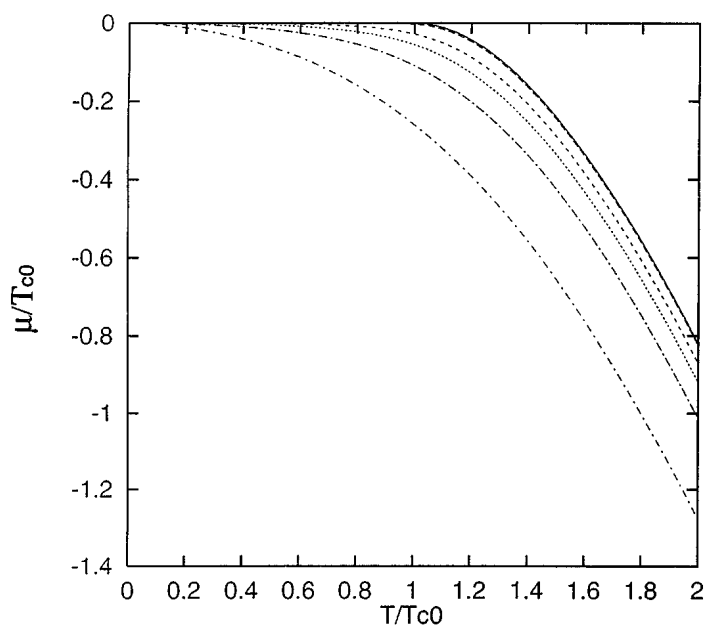

FIG. 1. Chemical potential of $\mathrm{CBG}$ as a function of temperature and magnetic field, $\omega_{H} / T_{c 0}=0,0.001,0.1,0.2$, 0.4, 1.0 (from top to bottom). 


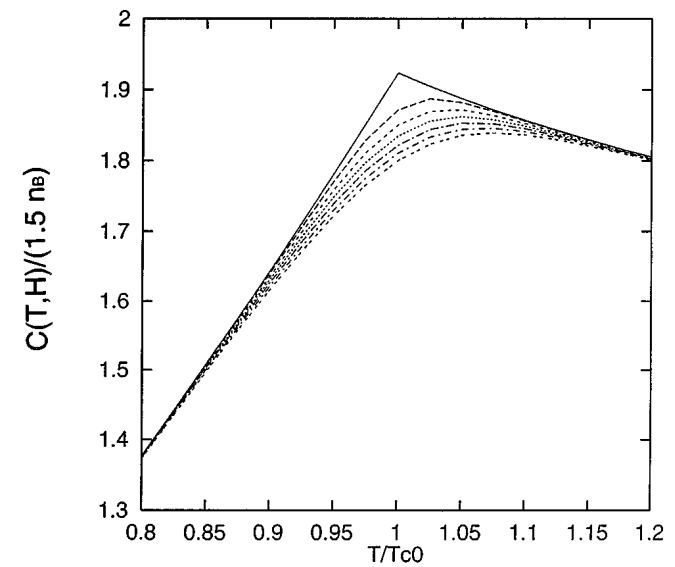

FIG. 2. Specific heat anomaly of the charged Bose gas for $\omega_{H} / T_{c 0}=0,0.004,0.008,0.012,0.016,0.020,0.024$.

lattice polarizability of the cuprates weakens the Coulomb repulsion between bosons. As a result its dimensional strength $r_{s}=16 \pi e^{2} m / \epsilon_{0}\left(4 \pi n_{B} / 3\right)^{1 / 3}$ becomes of the order of unity or less even for heavy carriers due to a very large static dielectric constant $\epsilon_{0} \gg 1$. In that case our theory becomes exact taking into account all quantum as well as the thermodynamic fluctuations [23]. A broadening of the Landau levels due to scattering has only a little effect on the specific heat anomaly. As an example, in the extreme case of a very large broadening one can choose a hybrid form of the zero and finite field DOS preserving the singular nature of the lowest Landau level as the only effect of the magnetic field,

$$
\rho(\epsilon, H) \approx \frac{m^{3 / 2} \sqrt{\epsilon}}{\sqrt{2} \pi^{2}}+\frac{m^{3 / 2} \omega_{H}}{2 \sqrt{2} \pi^{2} \sqrt{\epsilon}},
$$

where we have used the invariance of Eq. (4) to shift the zero of energy by $\omega_{H} / 2$. If this form of DOS is

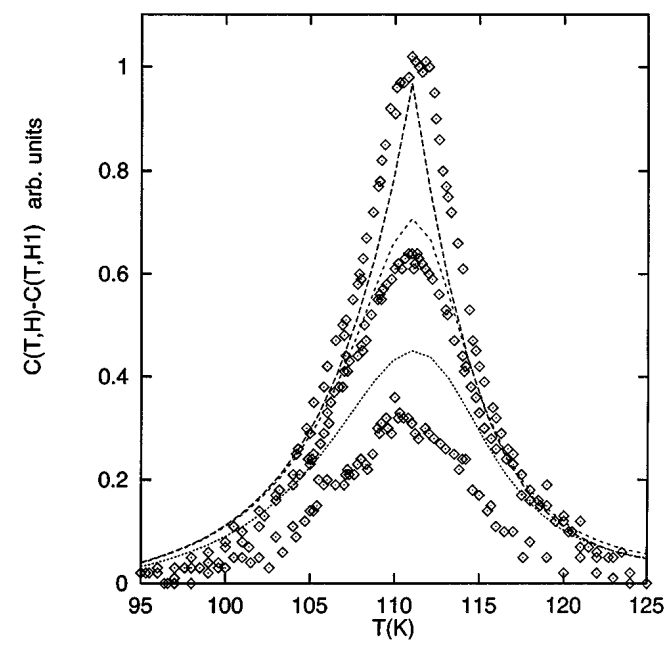

FIG. 3. Change in the specific heat of CBG with an applied magnetic field (different lines correspond to $H=0,0.52 T$ and $2 T$, respectively) compared with the experiment [21] for $\mathrm{Hg}-1223$. used to numerically calculate the specific heat, all results including the shape of the anomaly remain practically unchanged.

On the other hand, the theory of the Bose-Einstein condensation of CBG yields a significant change of the condensation temperature in a magnetic field if $T_{c} \tau \gg 1$, where $\tau$ is the scattering time for zero energy excitations. Without any broadening of the Landau levels $T_{c}$ drops to zero at any magnetic field as mentioned above. If the collision broadening of the Landau levels is taken into account, the resistive critical field is determined as [11]

$$
H_{c 2}(T)=H_{d}\left(t^{-1}-t^{1 / 2}\right)^{3 / 2},
$$

where $H_{d} \sim 1 /\left(T_{c} \tau\right)^{1 / 2}$ is a constant and $t=T / T_{c 0}$. This equation fits well several independent resistivity measurements $[6-8,19]$.

In conclusion, we have discussed the effect of the magnetic field on the specific heat of high- $T_{c}$ cuprates contrasting with that on the resistive transition. We have presented a reasoned argument that the specific heat anomaly which is observed in finite fields is, in fact, not the result of a superconducting phase transition. To substantiate our claim we have calculated the specific heat for a noninteracting charged Bose gas in a magnetic field. In this system there is no Bose condensation in a magnetic field, and yet there is still the characteristic maximum in the specific heat. We believe that the present study provided a good qualitative understanding of the startling experimental result $[1,20-22]$. We obtained a quantitative fit of our simplified model of weakly interacting charged bosons to the experimental shape. We explained this fact by the robustness of the calculated specific heat to the broadening of the Landau levels. The specific heat anomaly owes its origin to a sharp change in the temperature dependence of the chemical potential near $T_{c 0}$, Fig. 1, which is a feature of any Bose liquid irrespective of the interaction strength. A similarity between the specific heat of cuprates in a magnetic field and that of $\mathrm{He}^{4}$ films on Vycor with the different coverage supports our conclusion.

Enlightening discussions with J. Annet, J. R. Cooper, J. P. Franck, R. Giles, A. Junod, D. Khmelnitskii, F. Kusmarsev, G. Kaye, J. W. Loram, J. Samson, and K. R. A. Ziebeck are highly appreciated. We are particularly grateful to A. Junod for drawing our attention to the similarity between the specific heat transitions in high- $T_{c}$ cuprates in a magnetic field and those in the superfluid $\mathrm{He}^{4}$ films. One of us (V. V. K.) acknowledges support of the Russian Foundation for Basic Research (Grant No. 97-02-16705).

*Present address: H. H. Wills Physics Laboratory, Tyndall Avenue, Bristol BS8 1TL, United Kingdom.

[1] A. Junod in Studies of High Temperature Superconductors, Vol. 19, edited by A. Narlikar (Nova Science, Commack, NY, 1996), p. 1; S. E. Inderhees et al., Phys. Rev. 
Lett. 60, 1178 (1988); J. W. Loram et al., Physica (Amsterdam) 162-164C, 498 (1988); R. A. Fisher et al., Phys. Rev. B 38, 11942 (1988); W. Schnelle et al., Physica (Amsterdam) 168C, 465 (1990).

[2] J. W. Loram et al., Philos. Mag. B 65, 1405 (1992).

[3] B. Bucher et al., Physica (Amsterdam) 167C, 324 (1990).

[4] M. D. Lan et al., Phys. Rev. 47, 457 (1993).

[5] A. P. Mackenzie et al., Phys. Rev. Lett. 71, 1238 (1993).

[6] M.S. Osofsky et al., Phys. Rev. Lett. 71, 2315 (1993); 72, 3292 (1994).

[7] A. S. Alexandrov, V. N. Zavaritsky, W. Y. Liang, and P. L. Nevsky, Phys. Rev. Lett. 76, 983 (1996).

[8] D. D. Lawrie et al., in Proceedings of the MOS'96 Conference, Karlsruhe [J. Low Temp. Phys. (to be published)].

[9] Yu.N. Ovhinnikov and V.Z. Kresin, Phys. Rev. B 52, 3075 (1995)

[10] A. S. Alexandrov and N.F. Mott, Rep. Prog. Phys. 57, 1197 (1994); Polarons and Bipolarons (World Scientific, Singapore, 1995).

[11] A. S. Alexandrov, Phys. Rev. B 48, 10571 (1993).

[12] A.S. Alexandrov and J. Ranninger, Solid State Commun. 81, 403 (1992).

[13] M. Takigawa, Phys. Rev. B 42, 243 (1990); H. Zimmerman et al., Physica (Amsterdam) 185-189C, 1145 (1991);
T. Machi et al., Physica (Amsterdam) 173C, 32 (1991).

[14] Z. Schlesinger et al., Physica (Amsterdam) 185-189C, 57 (1991).

[15] D. M. King et al., J. Phys. Chem. Solids 56, 1865 (1996).

[16] A. S. Alexandrov, Physica (Amsterdam) 182C, 327 (1991); J. Low Temp. Phys. 87, 721 (1992).

[17] A. S. Alexandrov, A.M. Bratkovsky, and N.F. Mott, Phys. Rev. Lett. 72, 1734 (1994); A. S. Alexandrov, V. V. Kabanov, and N. F. Mott, ibid. 77, 4796 (1996).

[18] A. S. Alexandrov and D. K. Ray, Philos. Mag. Lett. 63, 295 (1991).

[19] A. S. Alexandrov, Phys. Rev. B 53, 2863 (1996).

[20] A. Junod et al., Physica (Amsterdam) 229C, 209 (1994).

[21] C. Marcenat, R. Calemczuk, and A. Carrington, in Coherence in High Temperature Superconductors, edited by G. Deutscher and A. Revcolevski (World Scientific, Singapore, 1995), and references therein.

[22] J. W. Radcliffe et al., J. Low Temp. Phys. 105, 903 (1996).

[23] A. S. Alexandrov, W. Y. Liang, and V.N. Zavaritsky, Phys. Rev. Lett. 78, 982 (1997).

[24] M. R. Schafroth, Phys. Rev. 100, 463 (1955).

[25] It was noticed in Ref. [12] that the carrier density determined from the value of the specific heat anomaly was about the same as the Hall density in cuprates. 